\title{
Qualidade de vida em pacientes adultos com paralisia cerebral
}

\section{Quality of life in adult patient with cerebral palsy}

\author{
Ana Paula Oliveira Mendes ${ }^{1}$, Anny Michelly Paquier Binha², Valéria Cassefo Silveira²
}

\section{RESUMO}

Paralisia cerebral (PC) é um grupo de desordens permanentes do desenvolvimento motor e postural que são atribuídas a distúrbios não progressivos que ocorreram no encéfalo em desenvolvimento. Diversos fatores podem interferir na qualidade de vida desses pacientes e com o aumento da longevidade é importante prevenir e intervir precocemente nos aspectos que comprometem a qualidade de vida. Objetivo: Verificar como os pacientes adultos com PC atendidos no ambulatório da AACD auto avaliam sua atual condição de saúde e correlacionar com alguns fatores que interferem na qualidade de vida dessa população. Método: Foi aplicado um questionário de triagem para avaliação de qualidade de vida dos pacientes via telefone (CDC HRQOL-4) e foi preenchido um protocolo de avaliação utilizando o prontuário. A coleta de dados foi realizada do dia 13 de março até 30 de junho de 2016. Realizada a análise estatística dos dados. Resultados: Foram atendidos 349 pacientes e 66 pacientes que obedeciam aos critérios de inclusão responderam ao questionário via telefone. A idade média geral foi de 26,5 anos. A divisão por sexo foi igual (50\%); a idade máxima foi de 52 anos. Houve predomínio de pacientes com diparesia espástica para ambos os sexos (57,5\% para mulheres e $54,5 \%$ para os homens). Entre as mulheres, $77,5 \%$ não estava praticando nenhuma atividade. No geral, $19,7 \%$ referiram alguma dor crônica e $31,8 \%$ tiveram queixa de alteração de humor nos últimos 30 dias. Na auto avaliação sobre a saúde em geral, $25,8 \%$ referiu estar excelente e apenas $10,6 \%$ regular. A maioria era solteiro(a), não tinha filhos e morava com a família (pais e irmãos). Conclusão: Os pacientes adultos com PC atendidos no ambulatório da AACD Ibirapuera são predominantemente adultos jovens, solteiros e que estão vivendo com seus familiares. Em geral, eles referem ter uma saúde muito boa ou excelente e menos de $20 \%$ convive com alguma dor crônica, mas esta não interfere nas atividades do cotidiano. Já em relação às alterações de humor, mais de um terço referiu ter estresse, tristeza ou ansiedade, mas também não tem forte influência na sua qualidade de vida.

Palavras-chave: Paralisia Cerebral, Qualidade de Vida, Adulto

\begin{abstract}
Cerebral palsy (CP) is a group of permanent motor and postural developmental disorders that are attributed to nonprogressive disorders that have occurred in the developing brain. Several factors can interfere with the quality of life of these patients and with the increase of longevity it is important to prevent and intervene early in the aspects that compromise the quality of life. Objective: To verify how the adult patients with CP treated at the AACD outpatient clinic self assess their current health condition and correlate with some factors that affect the quality of life of this population. Method: A screening questionnaire was applied to assess patients' quality of life via telephone (CDC HRQOL-4) and an evaluation protocol was completed using the medical record. Data collection was performed from March 13 to June 30, 2016. Statistical analysis of the data was performed. Results: 349 patients were treated and 66 patients who met the inclusion criteria answered the questionnaire via telephone. The overall average age was 26.5 years. The division by gender was equal (50\%); the maximum age was 52 years old. There was a predominance of patients with spastic diparesis for both sexes (57.5\% for women and $54.5 \%$ for men). Among women, $77.5 \%$ were not practicing any activity. Overall, $19.7 \%$ reported some chronic pain and $31.8 \%$ complained of mood swings in the last 30 days. In the self-rated general health, $25.8 \%$ reported being excellent and only $10.6 \%$ fair. Most were single, had no children and lived with their family (parents and siblings). Conclusion: Adult CP patients seen at the AACD Ibirapuera outpatient clinic are predominantly young, single adults living with their families. In general, they report very good or excellent health and less than $20 \%$ live with some chronic pain, but it does not interfere with daily activities. Regarding mood changes, more than one third reported having stress, sadness or anxiety, but that they have no strong influence on their quality of life.
\end{abstract}

\footnotetext{
Federal de São Paulo - UNIFESP.

${ }^{2}$ Médico Fisiatra, Associação de Assistência à
}

\section{Correspondência}

Anny Michelly Paquier Binha

Recebido em 06 Outubro 2017.

Aceito em 30 Outubro 2017.

\section{Como citar}

Mendes APO, Binha AMP, Silveira VC. Qualidade de vida em pacientes adultos com paralisia cerebral. Acta Fisiatr. 2018;25(2):49-53.

DOI: 10.11606/issn.2317-0190.v25i2a162559
Keywords: Cerebral Palsy, Quality of Life, Adult 


\section{INTRODUÇÃO}

Os indivíduos com paralisia cerebral (PC), por definição, têm seu desenvolvimento motor e postural alterado permanentemente por uma lesão não progressiva no encéfalo ainda imaturo. Esses distúrbios motores podem ser acompanhados de alterações da sensibilidade, percepção, cognição, comunicação e comportamento, por síndromes convulsivas e problemas musculoesqueléticos secundários. ${ }^{1}$

Estes são, portanto, fatores importantes que podem comprometer a qualidade de vida do indivíduo adulto com PC e que devem ser tratados quando possível. O termo qualidade de vida foi empregado pela primeira vez na década de 1960 e desde então vem sendo aplicado em diversos estudos da área da saúde, ciências sociais e filosofia. $\mathrm{O}$ desenvolvimento tecnológico trouxe uma grande preocupação com relação ao novo padrão de vida adotado principalmente na área urbana e a avaliação subjetiva da qualidade de vida se tornou uma ferramenta interessante para se estudar o impacto no estado de saúde. A mensuração da qualidade de vida serve também como um indicador da qualidade dos serviços oferecidos e das intervenções realizadas nessa população. $^{2}$

Estudos que investigaram a satisfação os adultos com paralisia cerebral com a própria saúde, observaram que ela é satisfatória ou excelente comparado a população em geral, e que aqueles que reportaram uma piora na qualidade que vida havia uma relação com a presença de dor e perdas funcionais, mas não com a gravidade da deficiência. ${ }^{3}$ No entanto a expectativa de vida dos pacientes com PC está aumentando e é evidente que a mobilidade, a funcionalidade e os problemas musculoesqueléticos já existentes irão sofrer alterações inerentes ao envelhecimento, oferecendo ainda mais dificuldades para esses indivíduos.

\section{OBJETIVO}

Verificar como os pacientes adultos com PC avaliam sua condição de saúde atual através de um questionário simples de quatro perguntas e correlacionar com alguns fatores que interferem na qualidade de vida dessa população.

\section{MÉTODO}

Este é um estudo transversal e observacional. Primeiro foi realizado uma revisão na literatura dos métodos de avaliação de qualidade de vida para a população em geral e elaborado um protocolo de avaliação que reúne as informações relevantes sobre cada paciente baseado em informações contidas no prontuário. $\mathrm{O}$ questionário escolhido para este estudo foi o "Healthy Days Questionnarie" (CDC HRQOL-4), que consiste de quatro perguntas simples e abrangentes. É um questionário validado em inglês e espanhol, e que permite mensurar de forma simples e rápida como o paciente avalia sua própria saúde física e mental, com o objetivo de realizar uma triagem desses pacientes. Este questionário breve (CDC HRQOL-4) está sendo amplamente usado nos sistemas de informação demográfica, pesquisas de prevenção e perfil de saúde da população. ${ }^{4}$

Desta forma o questionário poderia servir como uma ferramenta a mais para identificar os indivíduos com maiores demandas de assistência à saúde e por consequência, um risco maior de comprometimento da qualidade de vida. Foi, então, realizado a tradução para o português das quatro perguntas. A pontuação de dias "não saudáveis" é gerada somando a segunda e a terceira pergunta, com um escore máximo de 30. A primeira e a última pergunta são analisadas de forma independente.

A coleta de dados foi realizada do dia 13 de março até 30 de junho de 2016. Para participar do estudo os pacientes deveriam preencher os seguintes critérios: ter idade maior que 18 anos; ser capaz de compreender e responder às perguntas do questionário por telefone sem ajuda de terceiros; aceitar e assinar o termo de consentimento livre e esclarecido. Os pacientes atendidos na clínica de PC adulto foram informados sobre o estudo e convidados a participar assinando o termo de consentimento livre e esclarecido no dia da consulta. Um único examinador aplicou o questionário via ligação telefônica e os demais dados do protocolo foram obtidos diretamente do prontuário eletrônico. Após esse período foi realizada a análise dos dados utilizando o teste de ANOVA e o teste de QuiQuadrado. $O$ estudo foi aprovado pelo comitê de ética e pesquisa da AACD.

\section{RESULTADOS}

Foram atendidos 349 pacientes, 66 pacientes obedeciam aos critérios de inclusão e responderam ao questionário via telefone; 283 foram excluídos por não preencherem os critérios de inclusão, não terem atendido ao telefone cadastrado na ficha de atendimento ou por dificuldade de compreender as perguntas feitas pelo examinador (Figura 1). Dos 66 pacientes avaliados havia 33 homens e 33 mulheres com idade de 18 a 52 anos e a média de 26,5 anos. Com relação ao tipo de PC houve predomínio de pacientes com diparesia espástica $(n=37)$ e hemiparesia $(n=17)$.

$\mathrm{Na}$ auto avaliação sobre a saúde em geral, $25,8 \%$ referiu estar excelente e apenas $10,6 \%$ regular. A Figura 2 mostra a auto avaliação de saúde para cada tipo de PC, sendo que nos três grupos a maioria considera ter saúde muito boa ou excelente e o grupo com hemiparesia foi o que mais teve insatisfação, ou seja classificou sua saúde como regular.

Avaliamos os pacientes também quanto o nível de atividade física ou terapêutica semanal: $62 \%$ não realizam nenhuma atividade, $18 \%$ realiza de 1 a 2 vezes por semana e $20 \%$ realiza

\begin{tabular}{|c|c|}
\hline Nome: & Idade: \\
\hline RG: & Data nascimento: \\
\hline \multicolumn{2}{|l|}{ Diagnóstico: } \\
\hline Procedência: & GMFCS: \\
\hline Sexo: & Estado civil: \\
\hline Escolaridade: & Ocupação: \\
\hline \multicolumn{2}{|c|}{$\begin{array}{l}\text { Social: } \\
\text { - Família, Sozinho, Esposo(a) e filhos, Instituição }\end{array}$} \\
\hline $\begin{array}{l}\text { Dor: } \\
\text { - Sim } \\
\text { - Localização: } \\
\text { - Não }\end{array}$ & $\begin{array}{l}\text { Alteração de humor ou estado emocional: } \\
\text { - Sim } \\
\text { - Não }\end{array}$ \\
\hline \multicolumn{2}{|c|}{ Unhealthy Days Questionnaire (CDC HRQOL-4) } \\
\hline \multicolumn{2}{|c|}{$\begin{array}{l}\text { 1. Como você acha que está a sua saúde em geral? } \\
\text { - Excelente, muito boa, boa, regular ou ruim? } \\
\text { 2. Nos últimos } 30 \text { dias, quantos dias a sua saúde física esteve regular ou ruim? Por exemplo: Dor, cansaço, mal-estar } \\
\text { 3. Nos últimos } 30 \text { dias, quantos dias a sua saúde mental ou emocional esteve regular ou ruim? Por exemplo: Estresse, } \\
\text { ansiedade, tristeza } \\
\text { 4. Nos últimos } 30 \text { dias, quantos dias você deixou de fazer as suas tarefas do dia a dia por motivo de saúde? }\end{array}$} \\
\hline
\end{tabular}




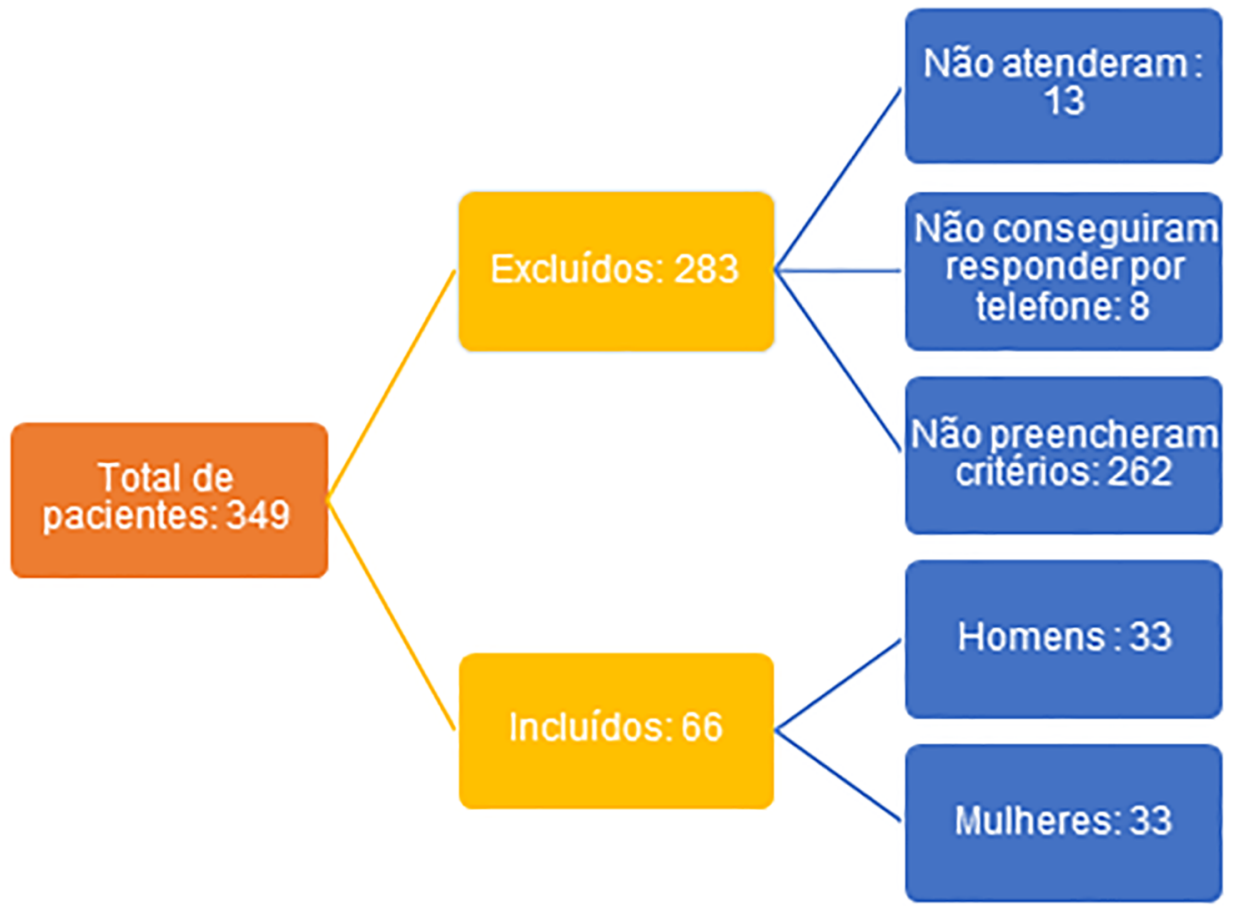

Figura 1. Pacientes incluídos de 3 a 5 vezes por semana. A Figura 3 mostra que os pacientes que avaliaram a saúde como excelente são os que praticam atividade física de 3 a 5 vezes na semana. Não houve diferença estatística significativa entre o numero de dias de atividade física semanal e a auto avaliação de saúde (valor de $p=0,8$ ).

$\mathrm{Na}$ última consulta médica, 19,7\% referiram alguma dor crônica e 31,8\% tiveram queixa de alteração de humor. Os mesmos pacientes que referiram dor crônica também tinham uma média de dias de atividade física semanal três vezes menos que a do grupo que não referiu dor. De acordo com a Figura 4 os pacientes com GMFCS II foram os que menos praticavam atividade física ou terapêutica $(16,7 \%)$ e os que mais realizavam foram os com GMFCS III (53,3\%).

A Figura 5 mostra a diferença do grupo com dor e o sem dor: o primeiro teve mais dias com algum problema físico, problema emocional e mais dias incapacitantes, que são as 3 ultimas

\section{TIPOS DE PC X AUTO-AVALIAÇÄO DE SAÚDE \\ $\because$ EXCELENTE $\square$ MUITOBOA $\square B O A \backsim R E G U L A R$}

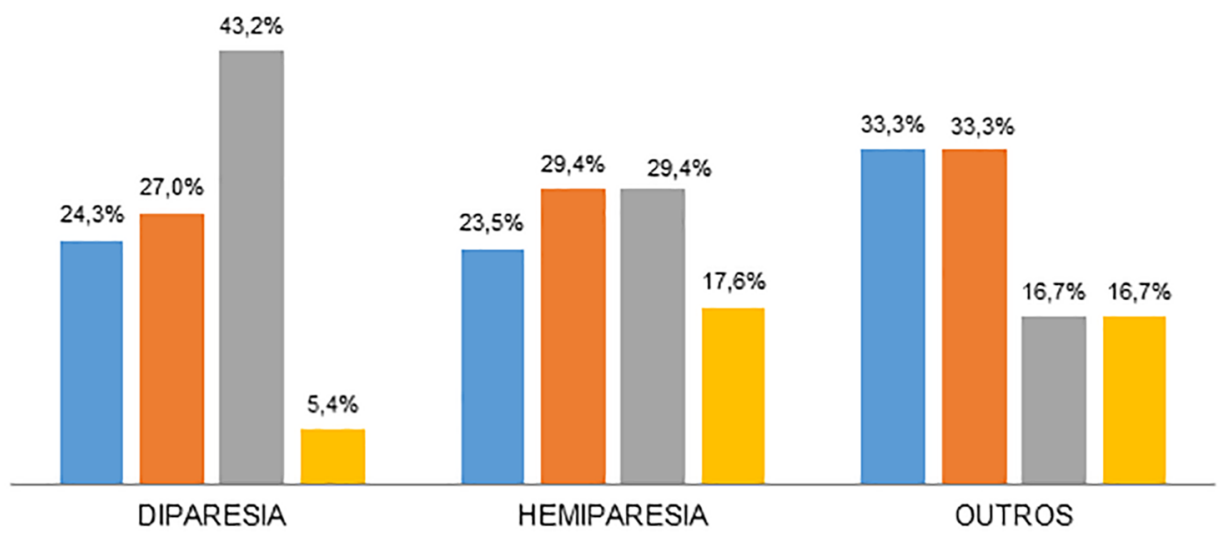

Figura 2. Auto avaliação de saúde em geral de acordo com o tipo de paralisia cerebral: diparesia, hemiparesia ou outros (atáxico, distônico, tetraparesia) perguntas do questionário. O escore do grupo com dor foi de 16,31 enquanto o sem dor foi de 3,63. Não houve diferença estatisticamente significativa entre a presença de dor, alteração de humor e o nível de GMFCS em relação a auto avaliação de saúde, com valores de $p>0,05$.

Os locais que os pacientes referiram ter dor crônica foram: lombar, joelho, quadril, pé, cervical e dois relataram ter cefaléia crônica (Quadro 1).

\section{DISCUSSÃO}

Este é um estudo transversal observacional que tem por objetivo saber como os pacientes adultos com PC atendidos no ambulatório da AACD na cidade de São Paulo avaliam sua condição geral de saúde e correlacionar com alguns fatores que interferem na sua qualidade de vida. No que diz respeito à característica da amostra dos 66 pacientes que completaram o questionário, eles são adultos jovens com média de 26,6 anos de idade, solteiros, que moram com seus familiares e sem diferença quanto a distribuição de gêneros. Esta amostra de pacientes se assemelha muito a outros estudos realizados com PC adultos. , $^{5,6}$

A maioria era procedente da cidade de São Paulo e não estava exercendo nenhuma atividade remunerada de trabalho. Dentro dos fatores avaliados neste estudo incluímos a frequência semanal de prática de atividade física ou terapêutica regular, como fisioterapia ou hidroterapia. Estudos anteriores que abordaram o tema qualidade de vida consideraram importantes a pratica regular de atividade física.7-11 $^{71}$

No nosso estudo os pacientes com GMFCS II são os que menos praticam atividade física ou terapêutica $(16,7 \%)$ e os que mais realizam são os com GMFCS III (53,3\%). Nooijen et al. ${ }^{7}$ encontrou que pacientes deambuladores jovens com PC são fisicamente menos ativos e tem um comportamento mais sedentário, principalmente os com diparesia espástica, do que a população em geral com a mesma idade.

Shkedy Rabani et al. ${ }^{8}$ ainda demonstram que adolescentes adultos jovens com PC ficam sedentários entre $82 \%$ e $96 \%$ do dia. Outro fator bem estudado na literatura é a dor crônica relatada por esses pacientes, variando de $14 \%$ a $73 \%$ de prevalência. ${ }^{12}$

Os pacientes no nosso estudo referiram ter mais dores relacionadas ao aparelho osteomuscular, sendo a lombalgia a mais prevalente, seguidos dos membros inferiores, sendo similar ao encontrado por Badia et al. ${ }^{13}$ A dor crônica tem um impacto negativo na qualidade de vida na população em geral. Nos 
Auto-avaliação de saúde $X$ atividade física semanal

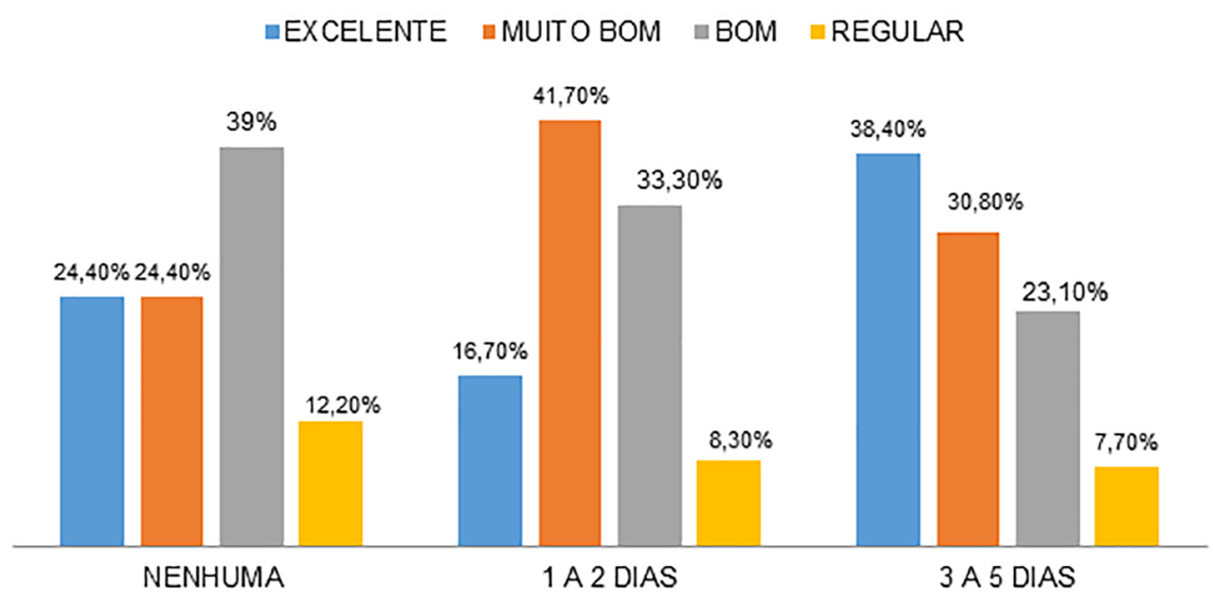

Figura 3. Auto avaliação de saúde de acordo com a frequência de atividade física ou terapêutica semanal

\section{Atividade Fisica / Terapêutica x GMFCS}

$$
\text { घSim } \square \text { Não }
$$

\begin{tabular}{c|cc} 
GMFCS V & $33,30 \%$ & $66,70 \%$ \\
\cline { 2 - 3 } GMFCS IV & $33,30 \%$ & $66,70 \%$ \\
\cline { 2 - 3 } & \multicolumn{2}{|c|}{$53,30 \%$} \\
GMFCS III & $36,70 \%$ \\
\cline { 2 - 3 } GMFCS II & $16,70 \%$ & $83,30 \%$ \\
\cline { 2 - 3 } GMFCS I & $36,70 \%$ & $63,30 \%$
\end{tabular}

Figura 4. Pratica de atividade física ou terapêutica semanal de acordo com o GMFCS

GRAU DE COMPROMETIMENTO DE SAÚDE NOS PACIENTE COM DOR

\author{
$\square$ DIAS DE SAUDE FISICAREGULAR OU RUIM \\ -DIAS DE SAUDE MENTAL / EMOCIONAL REGULAR OU RUIM \\ $=D I A S$ INCAPACITANTES \\ -ESCORE (DIAS "NÃO SAUDÁVEIS")
}

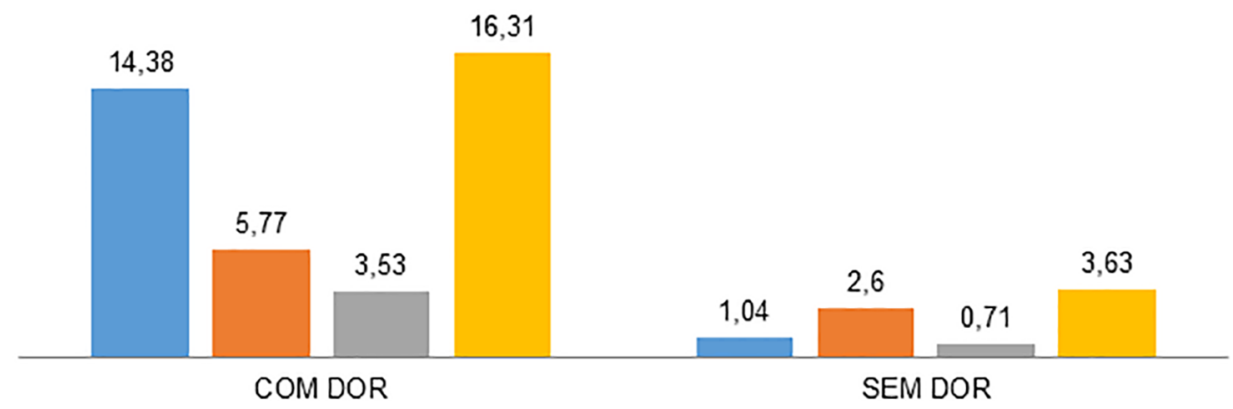

Figura 5. Grau de comprometimento da saúde física e mental nos pacientes com dor crônica pacientes com deficiência física, reconhecer e tratar adequadamente a dor pode ser um desafio, mas que precisa ser encarado como uma prioridade pelos médicos que acompanham estes pacientes..$^{12-14}$

No nosso estudo encontramos que os pacientes com dor tiveram mais dias incapacitantes para realização das atividades da vida diária e também eram os que tinham mais queixas emocionais, principalmente relacionadas a estresse e ansiedade. Outros estudos realizados com crianças com PC encontraram também que a intensidade da dor está relacionada ainda com mudanças no comportamento e problemas emocionais. ${ }^{14}$ Mais estudos precisam ser feitos para ver se a abordagem precoce da dor na infância tem interferência nos fatores psicológicos e comportamentais na idade adulta.

Por fim, o envelhecimento da população com PC já é uma realidade e a equipe multidisciplinar tem agora o desafio de dar mais qualidade de vida a esses pacientes. Utilizar instrumentos de triagem para identificar quais pacientes precisam de mais atenção da equipe pode ser interessante para instituições de referência em paralisia cerebral. Não devemos esquecer, no entanto, que esses pacientes também estão sujeitos a ter as doenças crônicas mais prevalentes na população em geral como hipertensão, diabetes mellitus e outras comorbidades que alongo prazo podem causar a piora funcional ${ }^{15}$ e por isso devem ser encaminhados aos especialistas capacitados para investigação e tratamento quando necessário.

Dentre as limitações do estudo podemos dizer que o número da amostra foi pequeno, o que pode talvez explicar a não significância estatística dos fatores estudados com a auto avaliação de saúde. O modo de aplicação do questionário via telefone também é limitante visto que esses pacientes podem ter dificuldades na fala e audição, dificultando a compreensão das perguntas realizadas.

\section{CONCLUSÃO}

A população estudada caracterizase por adulto jovem, solteiros, morando com a família e sem filhos, deambuladores comunitários independentes, com diparesia espástica. A maioria relatou ter uma saúde muito boa ou excelente e não referiu ter dor crônica ou alteração de humor. 
Quadro 1. Local da dor nos pacientes com PC

\begin{tabular}{|ll|}
\hline Local da dor & N \\
\hline Lombar & 8 \\
\hline Joelhos & 5 \\
Quadril & 2 \\
\hline Pés & 2 \\
\hline Cervical & 1 \\
\hline Cefaléia & 2 \\
\hline
\end{tabular}

\section{REFERÊNCIAS}

1. Rosenbaum $P$, Paneth $N$, Leviton A, Goldstein M, Bax $M$, Damiano $D$, et al. A report: the definition and classification of cerebral palsy April 2006. Dev Med Child Neurol Suppl. 2007;109:8-14.

2. Fleck MPA, Leal OF, Louzada $S$, Xavier $M$, Chachamovich E, Vieira G, et al. Desenvolvimento da versão em português do instrumento de avaliação de qualidade de vida da OMS (WHOQOL-100). Rev Bras Psiquiatr. 1999; 21(1):19-28. DOI: https://doi. org/10.1590/S1516-44461999000100006

3. Turk MA. Health, mortality, and wellness issues in adults with cerebral palsy. Dev Med Child Neurol 2009;51(S4):24-9. DOI: https://doi.org/10.1111/ j.1469-8749.2009.03429.x

4. Moriarty DG, Zack MM, Kobau R. The Centers for Disease Control and Prevention's Healthy Days Measures population tracking of perceived physical and mental health over time. Health Qual Life Outcomes. 2003;1:37. DOI: https://doi.org/10.1186/1477-7525-1-37
5. Binha AMP, Silveira VC. Avaliação clínico epidemiológica de adultos com paralisia cerebral: estudo retrospectivo. Med Reabil 2008; 27(3):76-81.

6. Margre ALM, Reis MGL, Morais RLS. Caracterização de adultos com paralisia cerebral. Rev Bras Fisioter. 2010;14(5):417-25. DOI: https://doi.org/10.1590/ S1413-35552010000500011

7. Nooijen CF, Slaman J, Stam HJ, Roebroeck ME, BergEmons RJ, Learn2Move Research Group. Inactive and sedentary lifestyles amongst ambulatory adolescents and young adults with cerebral palsy. J Neuroeng Rehabil. 2014;11:49. DOI: https://doi. org/10.1186/1743-0003-11-49

8. Shkedy Rabani A, Harries N, Namoora I, Al-Jarrah MD, Karniel A, Bar-Haim S. Duration and patterns of habitual physical activity in adolescents and young adults with cerebral palsy. Dev Med Child Neurol. 2014;56(7):673-80. DOI: https://doi. org/10.1111/dmcn.12394
9. Nakamura PM, Teixeira IP, Smirmaul BP, Sebastião E, Papini CB, Gobbi S, et al. Health related quality of life is differently associated with leisure-time physical activity intensities according to gender: a cross-sectional approach. Health Qual Life Outcomes. 2014;12:98. DOI: https://doi.org/10.1186/1477-7525-12-98

10. Brown DR, Carroll DD, Workman LM, Carlson SA, Brown DW. Physical activity and health-related quality of life: US adults with and without limitations. Qual Life Res. 2014;23(10):2673-80. DOI: https://doi. org/10.1007/s11136-014-0739-z

11. Luncheon C, Zack M. Health-related quality of life and the physical activity levels of middle-aged women, California Health Interview Survey, 2005. Prev Chronic Dis. 2011;8(2):A36.

12. Inoue $\mathrm{S}$, Kobayashi $\mathrm{F}$, Nishihara $\mathrm{M}$, Arai $\mathrm{YC}$, Ikemoto T, Kawai T, et al. Chronic Pain in the Japanese Community--Prevalence, Characteristics and Impact on Quality of Life. PLoS One. 2015;10(6):e0129262. DOI: https://doi.org/10.1371/journal.pone.0129262

13. Badia $M$, Riquelme I, Orgaz B, Acevedo R, Longo $E$, Montoya P. Pain, motor function and health-related quality of life in children with cerebral palsy as reported by their physiotherapists. BMC Pediatr. 2014;14:192. DOI: https://doi.org/10.1186/1471-2431-14-192

14. Yamaguchi R, Nicholson Perry K, Hines M. Pain, pain anxiety and emotional and behavioural problems in children with cerebral palsy. Disabil Rehabil. 2014;36(2):125-30. DOI: https://doi.org/10.3109/0963 8288.2013.782356

15. Peterson MD, Ryan JM, Hurvitz EA, Mahmoudi E. Chronic Conditions in Adults With Cerebral Palsy. JAMA 2015;314(21):2303-5. DOI: https://doi.org/10.1001/ jama.2015.11025 